\section{Jaundice as a presentation of heart failure}

As many as $55 \%$ of 42 patients with severe congestive cardiac failure $(\mathrm{CCF}$ ) (characterized by a cardiac index of $1.5 \mathrm{~L} /$ $\mathrm{min} / \mathrm{m}^{2}$ or less) may have raised serum alkaline phosphatase, ${ }^{1}$ yielding a prevalence of this liver enzyme derangement twice that documented by van Lingen et al. (September 2005 $\left.J R S M^{2}\right)$. Accordingly, jaundice and/or serum alkaline phosphatase hotline would be more informative than an exclusively jaundice hotline service for elucidating the distinguishing features (if any) of those patients in whom CCF co-exists with potentially treatable choledocholithiasis. This scenario is especially likely in the elderly, in whom the age-related nature of the two disorders ${ }^{3,4}$ makes it increasingly likely that they will co-exist by pure chance; the confusion between the two disorders is compounded when co-prescription of warfarin is complicated either by idiosyncratic warfarin-related cholestasis ${ }^{5}$ or by anticoagulant-related haemobilia. ${ }^{6}$

I agree that correct evaluation of jugular venous pressure (JVP) is crucial to the differential diagnosis between CCFrelated jaundice and other causes of jaundice. [I would add that the time is long overdue for non-cardiology internists to abandon the so-called 'JVP raised to $2 \mathrm{~cm}$ ' calibration so widely prevalent in the clerking of CCF patients]. However, this is not the whole story. The biochemical stigmata of CCF-related jaundice can be indistinguishable from those of choledocholithiasis, ${ }^{1}$ justifying a vigilant awareness of the possibility of the co-existence of the two disorders in every CCF patient in whom serum alkaline phosphatase of hepatic origin is elevated to twice or more of the upper limit of normal, irrespective of the severity of jaundice, and, possibly, even regardless of whether or not the patient is jaundiced. ${ }^{7}$

\section{O M P Jolobe}

Retired geriatrician

\section{REFERENCES}

1 Kubo SH, Walter BA, John DHA, Clark M, Cody RJ. Liver function abnormalities in chronic heart failure. Arch Intern Med 1987; 147:1227-30

2 Van Lingen R, Warshow U, Dalton HR, Hussaini SH. Jaundice as a presentation of heart failure. J R Soc Med 2005;98:357-9

3 McKee PA, Castelli WP, McNamara PM, et al. The natural history of congestive heart failure: the Framingham Study. $N$ Engl $J$ Med 1971;285:1441-6

4 Herman RE. The specrum of biliary stone disease. Am J Surg 1989;158: $171-3$

5 Adler E, Benjamin SB, Zimmerman HJ. Cholestatic hepatic injury related to warfarin exposure. Arch Intern Med 1986;146:1837-9

6 Jolobe OMP. Haemobilia: a possible cause of jaundice in patients receiving anticoagulants [Letter]. J Hepatol 1995;22:597-6

7 Jones SN, Askew CM, Beynon CPJ, Croker JR. Isolated elevation of serum alkaline phosphatase in biliary disease in the elderly. Postgrad Med J 1982;58:85-6

\section{Sir James Crichton-Browne}

In his biographical note on Sir James Crichton-Browne that John Hughlings Jackson was apprenticed to Thomas Laycock. In fact, Hughlings Jackson was apprenticed to Mr William Charles Anderson, a prosperous physician in the city of York. ${ }^{2}$ Laycock was Lecturer at the York Medical School, where he taught Hughlings Jackson the principles and practice of medicine. It was as a medical student that Hughlings Jackson was first exposed to Laycock's ideas about the reflex function of the brain, concepts that had an important influence on Jacksonian neurology. ${ }^{3}$

\section{George K York}

David A Steinberg

The Såa Institute, 21201 Ostrom Road, Fiddletown, CA 95629, USA

\section{REFERENCES}

1 Jellinek EH. Sir James Crichton-Browne (1840-1938): pioneer neurologist and scientific drop-out. J R Soc Med 2005;98:428-30

2 Ms 8241/18, p.58, No.7. In: The Society of Apothecaries' Court of Examiners' Candidates' Entry Book (1855-1858). London: Guildhall Library

3 Greenblatt SH. The major influences on the early life and work of John Hughlings Jackson. Bull Hist Med 1965;39:346-76

\section{The future of the printed journal}

The editorial about the impact of the information technology on the evolution of medical practice (September $2005 J_{R S} M^{1}$ ) is thought provoking. The editor seemed to end his note on somber tones regarding the future of printed medical journals.

As one who wrote about the future of electronic journals in $1996,{ }^{2}$ I am convinced that paper journals are here to stay despite open access publishing and other torments. This assertion is not based on data, but is more linked to the romance the human species has had with the rustling of paper since the middle ages. The thrill one gets while handling the crisp paper and turning a page over is largely unstudied. On a practical level, computer screens still have several limitations including portability. The present generation of professionals has not been trained in the 'all-by-computer-from-the-very-beginning' mode. The degree of access throughout wide sections of the world population will determine the growth of electronic medical publishing in the future.

Personally, I still prefer my paper copy of the Journal of The Royal Society of Medicine that comes in the unmistakable package than the online subscription!

\section{P Dileep Kumar}

Port Huron, Ml 48060, USA. E-mail: PDKumar@aol.com

\section{REFERENCES}

1 Abbasi K. Simplicity and complexity in health care: what medicine can learn from Google and iPod. J R Soc Med 2005;98:389

2 Kumar PD. The future for electronic journals. J R Soc Med 1996;89: 662 